\title{
Multistatic Radar Imaging via Decentralized and Collaborative Subspace Pursuit
}

\author{
Gang Li \\ EE Department \\ Tsinghua University \\ Beijing, China \\ gangli79.ee@gmail.com
}

\author{
Pramod K. Varshney \\ EECS Department \\ Syracuse University \\ Syracuse, NY, United States \\ varshney@syr.edu
}

\author{
Yimin D. Zhang \\ Center for Advanced Communications \\ Villanova University \\ Villanova, PA, United States \\ yimin.zhang@villanova.edu
}

\begin{abstract}
The task of multistatic radar imaging can be converted to the problem of jointly sparse signal recovery. In this paper, the algorithm named decentralized and collaborative subspace pursuit (DCSP) is utilized in multistatic radar systems to obtain a high-resolution image. By embedding collaboration among radar nodes and fusion strategy into each iteration of the standard subspace pursuit (SP) algorithm, DCSP is capable of providing satisfactory image even if some radar nodes suffer from relatively low signal-to-noise ratios (SNRs). Compared to the existing algorithms based on linear programming, DCSP has much lower computational complexity at the cost of increased communication overhead in the radar network.
\end{abstract}

Keywords-Multistatic radar imaging; compressive sensing; subspace pursuit.

\section{INTRODUCTION}

A multistatic radar with spatially dispersed transmitters and receivers contains multiple diverse monostatic radar and bistatic radar components with a common area of coverage. Compared to conventional monostatic radar with a co-located pair of transmitter and receiver, multistatic radars offer advantages in terms of spatial diversity and robustness to jamming. Examples of practical multistatic radar systems include the TechSAT 21 constellation [1], the Interferometric Cartwheel [2], and the multistatic ground-penetrating radar [3].

In this paper, we consider the task of multistatic radar imaging. The traditional algorithms of multistatic radar imaging are based on coherent processing of all receiver outputs [4][5]. The spatial diversity of multistatic radar increases the equivalent aperture and, therefore, the coherent processing of data acquired by a multistatic radar yields high imaging resolution and improves the signal-to-noise ratio (SNR). However, the multistatic configuration leads to different range-Doppler histories of the same target for each transmitter-receiver pair [6]. This causes the difficulty of coherent processing of multistatic radar data. In addition, coherent processing also requires a stable phase between the oscillators within the observation period [6]. Furthermore, when the aspect angles of the multiple radar nodes are

The work of G. Li was supported by the National Natural Science Foundation of China under Grant 41271011, the Program for New Century Excellent Talents in University under Grant NCET-11-0270 and the Tsinghua University Initiative Scientific Research Program. The work of P. K. Varshney was supported by National Science Foundation Award No. 1307775. significantly different, the data acquired at all the receivers cannot be coherently accumulated due to the angle-dependent scattering characteristics of the targets.

Recently, compressive sensing (CS) theory has been applied to radar imaging [7][8], since many radar imaging tasks can be posed as finding sparse solutions to underdetermined linear problems. In particular, the applications of CS in multistatic radar are introduced in [9]-[11]. The most attractive characteristic of CS in multistatic radar imaging applications is that a high-resolution image can be obtained without the requirement of coherent processing. Moreover, the benefits provided by CS-based imaging are more pronounced in some scenarios where the number of measurements is limited due to practical constraints. Most of the existing algorithms of multistatic radar imaging are based on linear programming (LP) and iterative Bayesian inference [9]-[11] and, therefore, computationally expensive.

In this paper, we utilize a greedy algorithm, decentralized and collaborative subspace pursuit (DCSP), to achieve highresolution imaging with a multistatic radar. In [12], DCSP was proposed for joint sparsity pattern recovery with Gaussian random dictionary matrices. In this paper, we evaluate the performance of DCSP with space-time Fourier dictionary matrices and applied it to multistatic radar imaging. One strength of DCSP is that the collaboration among radar nodes at each iteration ensures the consistency of the sparse structures of all local images. This is useful to remove artifacts from the fusion result of all the local images. Experimental evaluations show that, compared to the LP-based algorithm and the standard subspace pursuit (SP) algorithm with no collaboration [13], our approach can improve the image quality with much lower computational complexity. This benefit comes at the cost of increased communication overhead in the radar network.

\section{SigNAL MODEL}

The two-dimensional geometry of a multistatic radar system is demonstrated in Fig. 1(a). Assume that the multistatic radar system is composed of $Q$ monostatic radar nodes that work at different frequency bands. Bistatic components are not considered here. The common area of coverage is discretized to $M \times N$ cells (pixels), and all the monostatic radar nodes follow the same rule of discretization. The center of the observed area is taken as the coordinate origin. There are $K$ cells with 
dominant reflectivity in the observed region, where $K \ll M N$. It should be pointed out that, $K$ may be slightly larger than the number of actual targets, because some targets may occupy multiple discretized cells of the observed area. Each radar node is equipped with an antenna array composed of $L$ elements along cross-range direction. Arrange the array elements at each radar node so that the center of the observed region is located along the normal of the array ${ }^{1}$. The antenna array can be replaced with a synthetic aperture generated by motion of the radar platform along the cross-range direction, which refers to the concept of multistatic synthetic aperture radar (SAR) [6][9][11]. All the radar nodes collaborate to obtain the highquality image by sharing the local processing results with each other.

Assume that the step-frequency waveform is used at every radar node. At the $q$-th radar node, the baseband echo reflected from the cell $\left(x_{m}, y_{n}\right)$ can be expressed as

$y_{q}(p, l)=\alpha_{q, m, n} \cdot \exp \left[-j \frac{4 \pi}{c}\left(\left(f_{c, q}+p \Delta f\right) r_{q, m, n}+f_{c, q} d_{q, l} \sin \theta_{q, m, n}\right)\right]$,

where $p(1 \leq p \leq P)$ and $l(1 \leq l \leq L)$ denote the temporal sample index and spatial sample index, respectively, $\alpha_{q, m, n}$ is the complex reflectivity of the cell $\left(x_{m}, y_{n}\right), f_{c, q}$ is the carrier frequency of the $q$-th radar, $c$ is the wave propagation speed, $d_{q, l}$ is the spacing between the transmitter antenna and the l-th receiver, $\Delta f$ is the frequency step size, $r_{q, m, n}=\left[\left(x_{q}-x_{m}\right)^{2}+\left(y_{q}-\right.\right.$ $\left.\left.y_{n}\right)^{2}\right]^{1 / 2}$ is the distance between the $q$-th radar node and the cell $\left(x_{m}, y_{n}\right),-\pi / 2 \leq \theta_{q, m, n} \leq \pi / 2$ is steering angle of the cell $\left(x_{m}, y_{n}\right)$ with respect to the normal of the array.

The received signal at the $q$-th radar node can be considered as the sum of echoes reflected from all the cells. In the presence of the receiver noise, (1) can be rewritten as

$$
\mathbf{y}_{q}=\mathbf{A}_{q} \mathbf{x}_{q}+\mathbf{e}_{q},
$$

where $\mathbf{e}_{q} \in \mathbb{C}^{P L \times 1}$ is the additive Gaussian white noise,

$$
\begin{gathered}
\mathbf{y}_{q}=\left[y_{q}(1,1), \cdots, y_{q}(p, l), \cdots, y_{q}(P, L)\right]^{T} \in \mathbb{C}^{P L \times 1}, \\
\mathbf{x}_{q}=\left[\alpha_{q, 1,1}, \cdots, \alpha_{q, m, n}, \cdots, \alpha_{q, M, N}\right]^{T} \in \mathbb{C}^{M N \times 1},
\end{gathered}
$$

$\mathbf{A}_{q} \in \mathbb{C}^{P L \times M N}$ is the space-time Fourier dictionary matrix and its element can be expressed as

$$
\begin{aligned}
& \mathbf{A}_{q}(P(l-1)+p, M(n-1)+m) \\
& =\exp \left[-j \frac{4 \pi}{c}\left(\left(f_{c, q}+p \Delta f\right) r_{q, m, n}+f_{c, q} d_{q, l} \sin \theta_{q, m, n}\right)\right] .
\end{aligned}
$$

When the sampling scheme and the discretization rule are given, the dictionary matrix $\mathbf{A}_{q}$ can be pre-established. The following observations are in order: 1$) \mathbf{x}_{q}$ has only $K$ nonzero entries, since there are $K$ cells with dominant reflectivity in the observed region; 2) $\left\{\mathbf{x}_{q}, q=1,2, \cdots, Q\right\}$ have the same sparsity pattern, since all the radar nodes follow the same strategy of

\footnotetext{
${ }^{1}$ It is noted that enforcing the center of the observed region to be located along the normal of each array is not a necessary requirement. This assumption is made in this paper solely for the convenience of formulation.
}
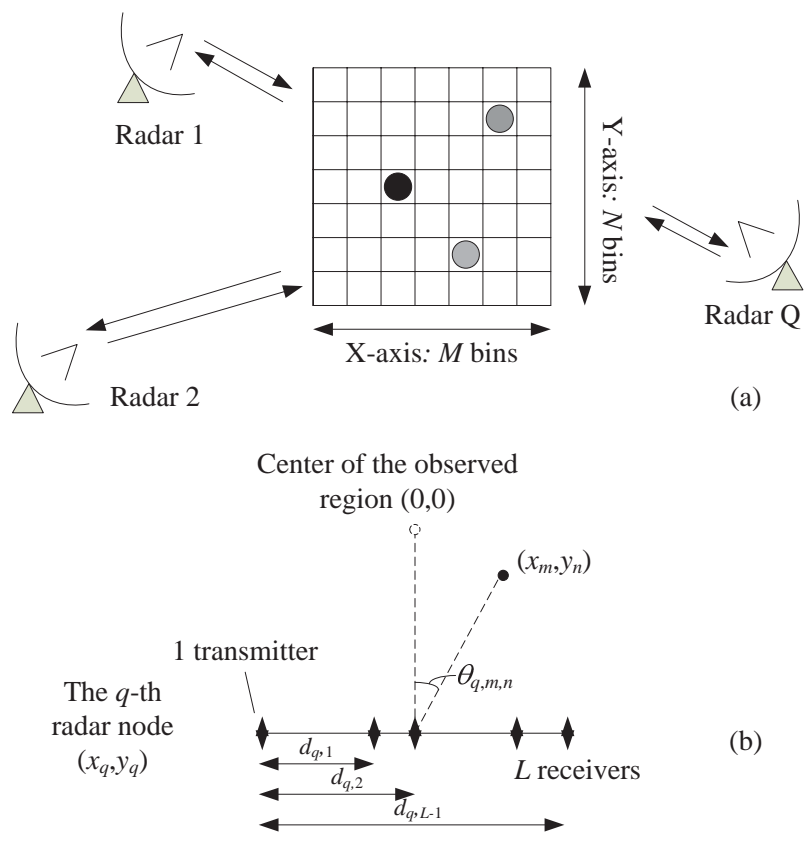

(b)

Fig. 1 Geometry of multistatic radar: (a) the radar network;

(b) the $q$-th radar node.

discretization of the observed area, and 3) the nonzero scattering coefficients $\mathbf{x}_{q}$ vary with the radar node index $q$ due to the variety of aspect angles and radar carrier frequencies. Thus, the task of multistatic radar imaging is converted to the problem of jointly sparse signal recovery. Define the common support set as $T=\left\{i: \mathbf{x}_{q}(i) \neq 0, i=1,2, \cdots, M N\right\}$. Once the support set $T$ is determined, the reflectivity $\left\{\mathbf{x}_{q}, q=1,2, \cdots, Q\right\}$ can be easily obtained by least squares estimation.

\section{DCSP FOR MULTISTATIC RADAR IMAGING}

In this section, we utilize the DCSP algorithm to achieve multistatic radar imaging. The DCSP algorithm is summarized in Algorithm 1. Some notations in Algorithm 1 are defined as follows.

- $\operatorname{proj}(\mathbf{y}, \mathbf{A})=\left[\mathbf{A}^{H} \mathbf{A}\right]^{-1} \mathbf{A}^{H} \mathbf{y}$ calculates the projection coefficients of a vector $\mathbf{y}$ onto the column space of matrix $\mathbf{A}$. Conjugate transpose is denoted by $(\cdot)^{H}$.

- $\operatorname{resid}(\mathbf{y}, \mathbf{A})=\mathbf{y}-\mathbf{A}\left[\mathbf{A}^{H} \mathbf{A}\right]^{-1} \mathbf{A}^{H} \mathbf{y}$ outputs the projection residual vector.

- $\quad$ max_ind $(\mathbf{y}, K)=\{K$ indices corresponding to the largest magnitude entries in vector $\mathbf{y}\}$.

- $\mathbf{A}(T)$ denotes a sub-matrix composed of the columns of $\mathbf{A}$ indexed by the set $T$.

- $\mathbf{y}(T)$ denotes a sub-vector composed of the entries of $\mathbf{y}$ indexed by the set $T$.

The basic idea of DCSP is to embed collaboration among radar nodes into each iteration of the standard SP algorithm. There are two major steps in DCSP: local estimation and fusion. In the local estimation phase, each radar node finds the subspace that the local received signal most probably lies in, as 
done in Steps 1 and 4. The vectors $\left\{\boldsymbol{\beta}_{q}^{v}, q=1,2, \cdots, Q\right\}$ record all the local estimates of the support set at the $v$-th iteration, and they are broadcast to the entire radar network. Fusion of all the local estimates of the support set is carried out by majority vote, as done in Steps 3 and 6. When the global recovery error reaches the minimum, iterations at all the radar nodes are terminated, and the fused image is obtained by averaging of all the local images, as done in Step 8.

\section{Algorithm 1 The DCSP algorithm}

Input: $K, \mathbf{y}_{q}, \mathbf{A}_{q}$ at the $q$-th radar node.

Initialization:

1) Let $\Gamma_{q}^{0}=$ max_ind $\left(\left|\mathbf{A}_{q}^{H} \mathbf{y}_{q}\right|, K\right)$. Define an $M N \times 1$ binary vector $\boldsymbol{\beta}_{q}^{0}$ as

$$
\boldsymbol{\beta}_{q}^{0}(i)=\left\{\begin{array}{lc}
1, & \text { if } i \in \Gamma_{q}^{0}, \\
0, & \text { otherwise. }
\end{array}\right.
$$

2) Send $\boldsymbol{\beta}_{q}^{0}$ to and receive $\boldsymbol{\beta}_{j}^{0}$ from the $j$-th radar node, for all $j \in\{1,2, \cdots, Q\} \backslash\{q\}$.

3) Let $\boldsymbol{\beta}^{0}=\sum_{q=1}^{Q} \boldsymbol{\beta}_{q}^{0}$. Find $T^{0}=\max \_$ind $\left(\boldsymbol{\beta}^{0}, K\right)$. Set the residual $\mathbf{r}_{q}^{0}=\operatorname{resid}\left(\mathbf{y}_{q}, \mathbf{A}_{q}\left(T^{0}\right)\right)$.

Iteration: at the $v$-th iteration $(v \geqslant 1)$

4) Let $\tilde{T}_{q}^{v}=T^{v-1} \cup \max \_i n d\left(\left|\mathbf{A}_{q}^{H} \mathbf{r}_{q}^{v-1}\right|, K\right)$ and $\Gamma_{q}^{v}=$ max_ind $\left(\left|\operatorname{proj}\left(\mathbf{y}_{q}, \mathbf{A}_{q}\left(\tilde{T}_{q}^{v}\right)\right)\right|, K\right)$. Define an $M N \times 1$ binary vector $\boldsymbol{\beta}_{q}^{v}$ by

$$
\boldsymbol{\beta}_{q}^{v}(i)=\left\{\begin{array}{lc}
1, & \text { if } i \in \Gamma_{q}^{v}, \\
0, & \text { otherwise. }
\end{array}\right.
$$

5) Send $\boldsymbol{\beta}_{q}^{v}$ to and receive $\boldsymbol{\beta}_{j}^{v}$ from the $j$-th radar node, for all $j \in\{1,2, \cdots, Q\} \backslash\{q\}$.

6) Let $\boldsymbol{\beta}^{v}=\sum_{q=1}^{Q} \boldsymbol{\beta}_{q}^{v}$. Find $T^{v}=\max \_$ind $\left(\boldsymbol{\beta}^{v}, K\right)$. Update the residual $\mathbf{r}_{q}^{v}=\operatorname{resid}\left(\mathbf{y}_{q}, \mathbf{A}_{q}\left(T^{v}\right)\right)$.

7) Send $\left\|\mathbf{r}_{q}^{v}\right\|_{2}^{2}$ to and receive $\left\|\mathbf{r}_{j}^{v}\right\|_{2}^{2}$ from the $j$-th radar node, for all $j \in\{1,2, \cdots, Q\} \backslash\{q\}$.

8) If $\sum_{q=1}^{Q}\left\|\mathbf{r}_{q}^{v}\right\|_{2}^{2} \geq \sum_{q=1}^{Q}\left\|\mathbf{r}_{q}^{v-1}\right\|_{2}^{2}$, let $T^{v}=T^{v-1}$ and stop the iteration. Define an $M N \times 1$ vector $\hat{\mathbf{x}}_{q}$ by

$$
\begin{aligned}
& \hat{\mathbf{x}}_{q}\left(T^{v}\right)=\left|\operatorname{proj}\left(\mathbf{y}_{q}, \mathbf{A}_{q}\left(T^{v}\right)\right)\right|, \\
& \hat{\mathbf{x}}_{q}(i)=0 \quad\left(i \notin T^{v}\right) ;
\end{aligned}
$$

Otherwise, let $v=v+1$ and return to Step 4 .

Output: The local imaging results $\left\{\hat{\mathbf{x}}_{q}, q=1,2, \cdots, Q\right\}$ and the fused image $\hat{\mathbf{x}}=\sum_{q=1}^{Q} \hat{\mathbf{x}}_{q} / Q$.

It should be pointed out that, the sparsity of the signal, $K$, needs to be pre-estimated, since it is used as an input parameter of DCSP. One way to roughly estimate $K$ is first employing Fourier-based imaging algorithms at a radar node and then broadcasting the estimated number of dominant targets to other radar nodes. An alternative approach is to set $\hat{K} \approx P L / 2$, i.e., half the number of measurements per radar node, which is large enough for DCSP to image all the dominant cells in most sparsity-driven imaging applications yet to ensure the image reconstructability.

\section{EXPERIMENTAL RESULTS}

In this section, we evaluate our approach via numerical experiments. The observed region is discretized as $30 \times 30$ cells (i.e., $M=N=30$ ) with cell size of $1 \mathrm{~m}$. Assume that there are $K=11$ cells with dominant reflectivity in the observed region. The multistatic radar system is composed of 4 monostatic radar nodes that have the same bandwidth $10 \mathrm{MHz}$ but different carrier frequencies $5 \mathrm{GHz}, 5.2 \mathrm{GHz}, 5.4 \mathrm{GHz}$ and $5.6 \mathrm{GHz}$. The SNRs at these radar nodes are $7 \mathrm{~dB}, 3 \mathrm{~dB}, 0 \mathrm{~dB}$ and -3dB, respectively, where $\mathrm{SNR}_{q} \triangleq\left\|\mathbf{A}_{q} \mathbf{x}_{q}\right\|_{2}^{2} /\left(P L \sigma^{2}\right)$ and $\sigma^{2}$ is the variance of the noise. The aspect angles of these radar nodes are $-45^{\circ}, 0^{\circ}, 20^{\circ}$ and $45^{\circ}$, respectively, leading to distinct entries of $\mathbf{x}_{q}$ for $q=1,2, \cdots, Q$. The fusion result of all the local scattering coefficients is considered as the ideal image, i.e., $\mathbf{x}_{\text {ideal }}=\sum_{q=1}^{Q}\left|\mathbf{x}_{q}\right| / Q$, which is shown in Fig. 2(a). With 81 measurement samples ( $P=9$ temporal samples and $L=9$ spatial samples) per radar node, the images provided by the LP algorithm, the standard SP algorithm with no collaboration, and the DCSP algorithm are shown in Figs. 2(b), 2(c), and 2(d), respectively. Images shown in Figs. 2(b), 2(c), and 2(d) are obtained by fusing all the local images and averaging over 10 trials, i.e., $\hat{\mathbf{x}}=\sum_{\text {trial }=1}^{10} \sum_{q=1}^{Q}\left|\hat{\mathbf{x}}_{q \text {,trial }}\right| /(10 Q)$. In each trial, the temporal and spatial samples per radar node are randomly and independently generated, which means that $\mathbf{A}_{q}$ varies with the radar index $q$ and the trial index. For the LP algorithm, each radar node independently solves for the local image via $L_{1}$ norm minimization, and then all the local images are fused to obtain the final imaging result. Such a framework is similar to that in [14]. For the standard SP algorithm, the local images are independently obtained via SP and then fused. In both algorithms, there is no collaboration among radar nodes before the solution processes at all the radar nodes are completed. Accordingly, errors of local images obtained at some nodes with low SNRs affect the quality of the fused image. As seen in Figs. 2(b) and 2(c), the images formed by these two algorithms suffer from some artifacts. Different from the above two algorithms, in DCSP we embed the collaboration among radar nodes into the iterative solution process. This corrects the local reconstruction errors of at each iteration and avoids error accumulation in subsequent iterations. As shown in Fig. 2(d), the DCSP well retrieves the scattering coefficients of the observed region without artifacts.

Next, we compare different algorithms in terms of the computational complexity per radar node. The complexity of the LP algorithm based on interior point method is $O\left[(P L)^{2}(M N)^{1.5}\right][13]$. The standard SP algorithm requires $O(K)$ iterations, and the complexity of each iteration is $O\left[P L\left(M N+K^{2}\right)\right]$. Thus, the complexity of the standard SP algorithm is $O\left[K P L\left(M N+K^{2}\right)\right]$. As stated in [12], thanks to the collaboration among nodes per iteration, the number of 


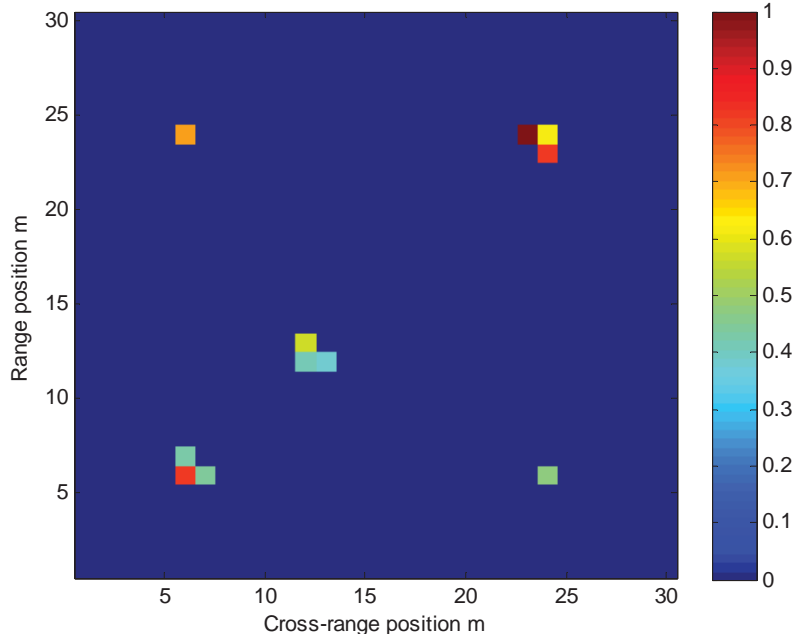

(a)

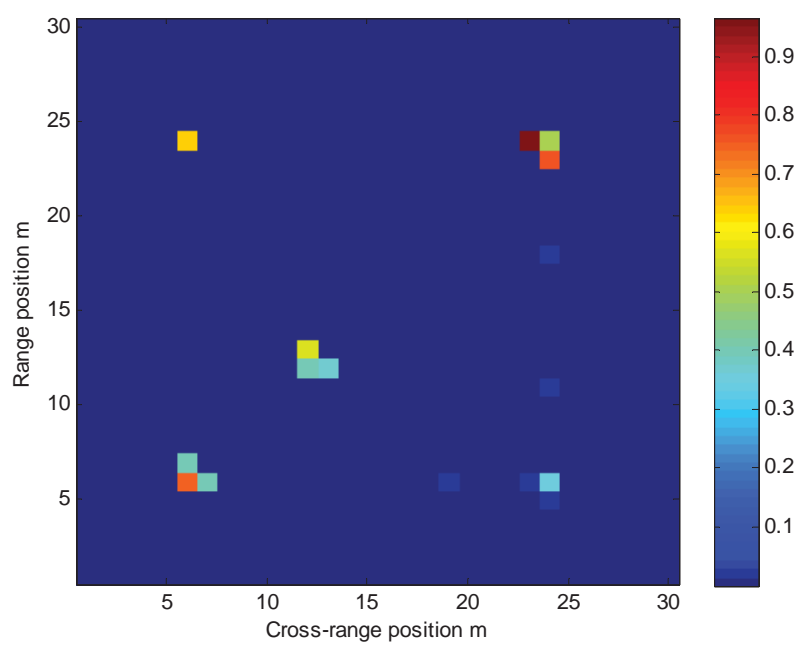

(b)

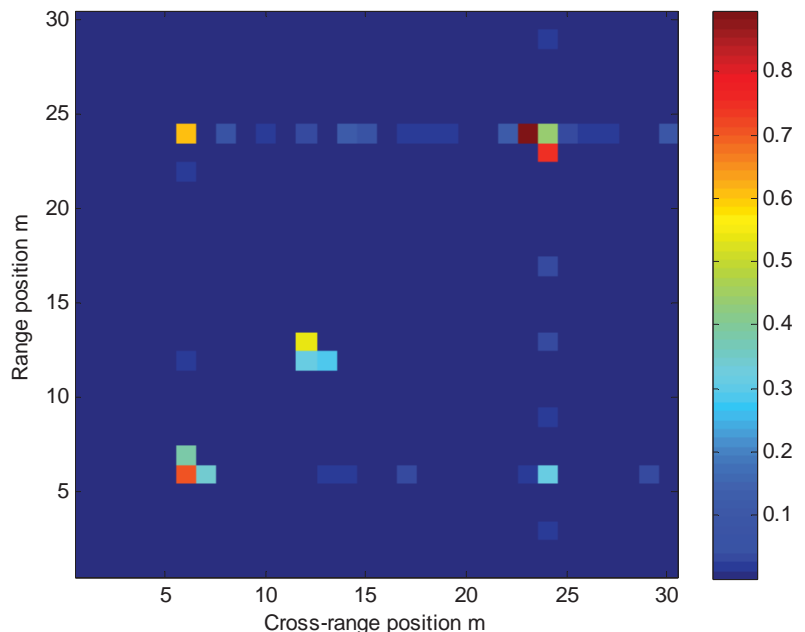

(c)

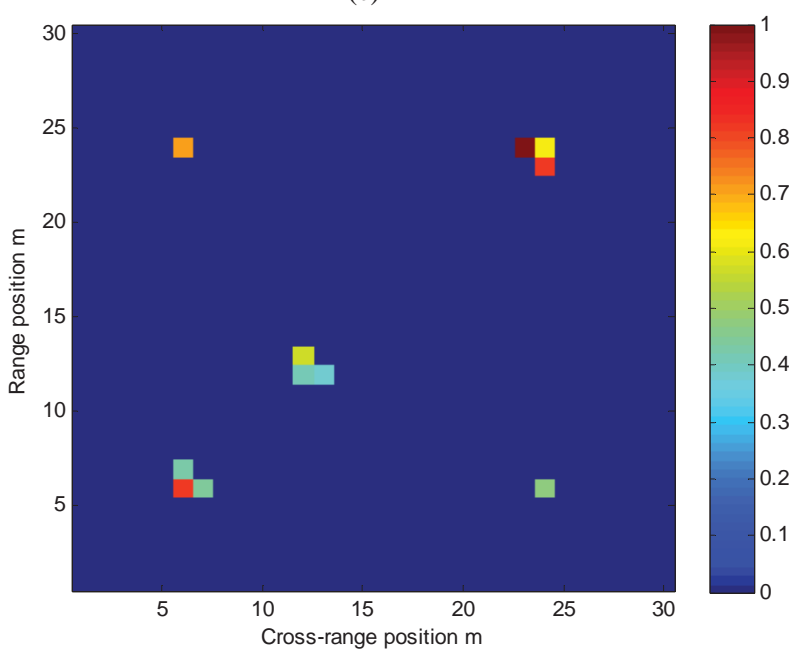

(d)

Fig. 2 Fused images. (a) True scattering coefficients, (b) Image obtained by linear programming, (c) Image obtained by the standard SP algorithm, (d) Image obtained by DCSP.

iterations of DCSP is $O(K)$. Moreover, the procedure at each iteration of DCSP is similar to that of SP. Therefore, the computational complexity of DCSP is also $O\left[K P L\left(M N+K^{2}\right)\right]$. The running times of the above three algorithms are estimated by averaging over 50 trials and listed in Table 1, which shows the computational efficiency of DCSP. All the results in Table 1 are obtained by using MATLAB R2011b with 64-bit operating system on a laptop with an Intel(R) Core(TM) i74600U CPU@2.10.GHz.

Table 1 Running times of different algorithms

\begin{tabular}{|c|c|c|c|}
\hline Algorithm & LP & SP & DCSP \\
\hline Running time (sec) & 2.39 & 0.04 & 0.06 \\
\hline
\end{tabular}

The above experiments show that, compared to the LPbased algorithm and the standard SP algorithm with no collaboration, the approach proposed in this paper can improve image quality with much lower computational complexity. It should be emphasized that this benefit comes at the cost of increased communication overhead in the radar network. The communication overhead can be considered proportional to the number of messages to be transmitted from all the radar nodes. For the LP-based algorithm and the standard SP algorithm with no collaboration, each one of the $Q$ radar nodes independently solves for the local $M N \times 1$ image and then broadcasts to the entire radar network. Thus, the communication overhead of these two algorithms is MNQ. At each iteration of DCSP, each radar node needs to share the $M N \times 1$ vector $\boldsymbol{\beta}_{q}^{v}$ and the local recovery error $\left\|\mathbf{r}_{a}^{\mathbf{v}}\right\|_{2}^{2}$ with others in the network, as described in Steps 1, 4 and 8 in Algorithm 1. As stated above, the number of iterations of DCSP is $O(K)$. Thus, the communication overhead of DCSP is $O(K)(M N+1) Q$, which is $O(K)$ times of that of the LP-based algorithm and the standard SP algorithm with no collaboration.

\section{CONCLUSION}

The task of multistatic radar imaging can be converted to the problem of jointly sparse signal recovery. From this 
observation, in this paper we modified an algorithm named DCSP for multistatic radar imaging. The basic idea of DCSP is to embed collaboration among radar nodes and fusion strategy into each iteration of the standard SP algorithm. The collaboration among radar nodes at each iteration of DCSP ensures the consistency of all the local estimates of the support set and, therefore, corrects the local image errors at those nodes with low SNRs. Compared to the LP-based algorithm and the standard SP algorithm with no collaboration, DCSP avoids the occurrence of artifacts and, therefore, improves the image quality. The computational complexity of DCSP is comparable with that of SP and much lower than that of LP-based algorithm. The improvement of the performance of DCSP comes from the cost of increased communication overhead in the radar network. Future work includes convergence analysis and extension to the case of extended targets.

\section{REFERENCES}

[1] M. Martin, P. Klupar, S. Kilberg, and J. Winter, "Techsat 21 and revolutionizing space missions using microsatellites," in Proceedings of the 15th American Institute of Aeronautics and Astronautics Conference on Small Satellites, Utah, 2001.

[2] D. Massonnet, "Capabilities and limitations of the interferometric cartwheel," IEEE Transactions on Geoscience and Remote Sensing, vol. 39, no. 3, pp. 506-520, Mar. 2001.

[3] T. Counts, A. C. Gurbuz, W. R. Scott, Jr., J. H. McClellan, and K. Kim, "Multistatic ground-penetrating radar experiments," IEEE Transactions on Geoscience and Remote Sensing, vol. 45, no. 8, pp. 2544-2553, Aug. 2007.

[4] G. Krieger, H. Gebert, and A. Moreira, "Unambiguous SAR signal reconstruction from nonuniform displaced phase center sampling,” IEEE Geoscice and Remote Sensing Letters, vol. 1, no. 4, pp. 260- 264, 2004.
[5] T. Varsolt, B. Yazici, and M. Cheney, "Wide-band pulse-echo imaging with distributed apertures in multi-path environments," Inverse Problems, vol. 24, no. 4, Aug. 2008.

[6] G. Krieger and A. Moreira, "Spaceborne bi- and multistatic SAR: potential and challenges," IEE Proceedings-Radar, Sonar and Navigation, vol. 153, no. 3, pp. 184-198, June 2006.

[7] L. C. Potter, E. Ertin, J. T. Parker, and M. Cetin, "Sparsity and compressed sensing in radar imaging," Proceedings of the IEEE, vol. 98, no. 6, pp. 1006-1020, June 2010.

[8] J. H. G. Ender, "On compressive sensing applied to radar," Signal Processing, vol. 90, no. 5, pp. 1402 - 1414, May 2010.

[9] I. Stojanovic, W. C. Karl, and M. Cetin, "Compressed sensing of monostatic and multi-static SAR," in Proceeding of Algorithms Synthetic Aperture Radar Imagery XVI, SPIE, Apr. 2009.

[10] N. S. Subotic, B. Thelen, K. Cooper, W. Buller, J. Parker, J. Browning, and H. Beyer, "Distributed RADAR waveform design based on compressive sensing considerations," in Proceedings of IEEE Radar Conference, pp. 1-6, Rome, Italy, May 2008.

[11] Q. Wu, Y. D. Zhang, M. G. Amin, and B. Himed, "Multi-Static Passive SAR Imaging Based on Bayesian Compressive Sensing," in Proceedings of Compressive Sensing Conference III, SPIE, May 2014.

[12] G. Li, T. Wimalajeewa, and P. K. Varshney, "Decentralized subspace pursuit for joint sparsity pattern recovery," in Proceedings of the IEEE International Conference on Acoustics, Speech and Signal Processing (ICASSP), Florence, Italy, May 2014.

[13] W. Dai and O. Milenkovic, "Subspace pursuit for compressive sensing signal reconstruction," IEEE Transactions on Information Theory, vol. 55, no. 5, pp. 2230-2249, May 2009.

[14] Z. Tian, E. Blasch, W. Li, G. Chen, and X. Li, "Performance evaluation of distributed compressed wideband sensing for cognitive radio networks," in Proceedings of IEEE International Conference on Information Fusion, Cologne, Germany, July 2008. 